ORIGINAL PAPER

\title{
EVALUATION OF DIFFERENT P 16 IMMUNOSTAINING METHODS AND THE PROGNOSTIC ROLE OF P16/KI-67 COMBINED EXPRESSION IN NON-MUSCLE INVASIVE BLADDER CANCERS
}

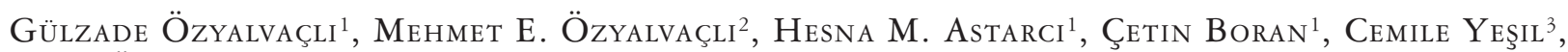 \\ Ugur Üyetürk ${ }^{2}$, Gülan Aktaş ${ }^{1}$
}

\begin{abstract}
${ }^{1}$ Department of Pathology, Medical Faculty, Abant Izzet Baysal University, Bolu, Turkey ${ }^{2}$ Department of Urology, Medical Faculty, Abant Izzet Baysal Universtiy, Bolu, Turkey ${ }^{3}$ Synevo Medical Laboratory, Ankara, Turkey
\end{abstract}

\begin{abstract}
There are many scoring methods evaluating the expression of p16 in the bladder immunohistochemically. In this study our aim was to determine an optimal p16 scoring method by discussing different staining methods related with p16 expression in bladder cancers and to establish the association of p16 and $\mathrm{Ki}-67$ expressions, alone or in combination, with recurrence and progression. Ninety patients undergoing their first transurethral resection for bladder cancer and newly diagnosed papillary urothelial carcinoma (pTa and pT1) were included in the study. Four different scoring methods were used for p16 (p16a, p16b, p16c, p16d). The patients were divided into two groups based on recurrence and progression. There was a statistically significant difference between recurrence and abnormal p16d staining $(\mathrm{p}=0.005)$. In other staining patterns of $\mathrm{p} 16$, there was no statistically significant difference in terms of recurrence or progression.In the multivariate logistic regression analysis, combined $\mathrm{Ki}-67 \geq 10$ and abnormal p16d staining was found to be the only independent predictive factor for recurrence $(\mathrm{OR}=2.26,95 \% \mathrm{CI}: 0.13-46.41, \mathrm{p}=$ $0.035)$ and no independent predictive factor for progression was found. Determining an adequate expression scoring by taking normal transitional epithelial staining pattern as a reference would be an objective approach in p16 evaluation. Moreover, it was found that evaluating p16d and $\mathrm{Ki}-67$ in combination would be significant in predicting recurrence in $\mathrm{pTa}$ and $\mathrm{pT} 1$ urothelial carcinomas.
\end{abstract}

Key words: bladder cancer, Ki-67, p16INK4A, progression, recurrence.

\section{Introduction}

Bladder cancer is a complex and multi-factorial cancer involving both environmental and genetic factors [1]. During diagnosis, nearly $75 \%$ of bladder cancers are superficial which are confined to the mucosa or lamina propria [2-4]. However, although superficial, nearly $30-80 \%$ of these tumors show recurrence and 1-50\% show progression [2-6]. Tumor diameter, number, grade and stage as used in rou- tine practice, may fail to determine the clinical course of these tumors most of the time [7]. Thus, there is a continuing need for new molecular and immunohistochemical markers to determine the clinical progression and treatment protocols in these patients.

As with many cancers, genetic mutations occurring in cell cycle proteins are frequently observed in bladder cancer. p16, a cyclin-dependent kinase inhibitor, is also considered to be a tumor suppressor gene. In many cancers, p16 is inactivated through muta- 
tion, methylation, or deletion. p16 acts at the G1-S checkpoint by increasing $\mathrm{Rb}$ protein levels, while loss of p16 causes inadequate cell proliferation through loss of $\mathrm{Rb}$ function [8-11]. Many studies have shown that inactivation of the $\mathrm{p} 16 /$ cyclin $\mathrm{D} 1 / \mathrm{Rb}$ pathway during oxidative stress plays an important role in the development of urothelial carcinoma (UC) in the bladder $[12,13]$. There are many scoring methods for evaluating the expression of p16 in the bladder and other tumors immunohistochemically; however, no standard scoring system or optimal p16 staining method is available.

$\mathrm{Ki}-67$ is a nuclear antigen expressed in the G1, S, and G2, but not the G0, phases of the cell cycle. Tumor recurrence and progression are significantly associated with the $\mathrm{Ki}-67$ index in non-muscle invasive bladder cancers [14-16].

The aim of this study was to determine the most appropriate p16 scoring method by comparing various staining methods related to p16 expression in bladder cancers and to assess the association between p16 and Ki-67 expression, alone or in combination, with recurrence and progression.

\section{Material and methods}

\section{Patient and tumor characteristics}

In total, 90 consecutive patients undergoing their first transurethral resection (TUR) for bladder cancer and newly diagnosed papillary UC (pTa and pT1) as a result of pathology between 2005 and 2013 were included. Approval for this study was obtained from the Ethics Committee of our university. Clinical data (age, gender, grade, stage, smoking, tumor size and number, presence of CIS, re-TUR, postoperative immunotherapy, and chemotherapy) of the patients and histopathological preparations were received from Abant Izzet Baysal University Urology and Pathology Department and evaluated by two pathologists (GO and MA) blinded to the patient characteristics. All tumors were graded in accordance with World Health Organization (WHO)/International Society of Urological Pathology (ISUP) (2004). Tumor stage was classified according to the Union for International Cancer Control (UICC) 2009 TNM classification.

\section{Patient follow-up}

Recurrence was defined as the relapse of UC of the bladder at any pathological stage after the initial surgery. Progress to a higher stage or metastasis, confirmed histopathologically, was deemed progression. Patients having carcinoma in situ, undergoing a second TUR, or receiving intracavitary chemotherapy and immunotherapy were excluded from the study.

\section{Immunohistochemistry}

Immunohistochemistry (IHC) was performed using a streptavidin-biotin-peroxidase technique (Bond fully integrated IHC and in situ hybridization (ISH) system; Leica) with a monoclonal antibody to p16 (Medaysis, 1 : 30 dilution) and Ki-67 (clone MM1; Leica). First, $5-\mu \mathrm{m}$ sections from paraffin wax-embedded samples were cut and placed on poly-L-lysine-coated slides, deparaffinized with xylene, and then rehydrated. For antigen retrieval, the slides were treated by microwave heating in citrate buffer $(\mathrm{pH}$ 6.0) for $10 \mathrm{~min}$. Next, $3 \%$ hydrogen peroxide was used to block endogenous peroxidase activity.

The sections were incubated with primary antibodies, including those against p16 and $\mathrm{Ki}-67$, for $1 \mathrm{~h}$ at room temperature. After washing in phosphate-buffered saline, the samples were incubated with a biotin-conjugated secondary antibody and then with a streptavidin-biotin system for $30 \mathrm{~min}$ at room temperature. The reactions were visualized by immersion of the specimens in diaminobenzidine tetrahydrochloride. The sections were counterstained with hematoxylin, then rinsed and mounted.

\section{Immunohistochemical scoring system}

While there was cytoplasmic p16 expression in tumors that exhibited strong staining, there was no cytoplasmic expression in p16-negative tumors. Although this staining was thought to be specific for p16, only nuclear staining was taken into account when scoring $\mathrm{p} 16$. To understand the staining pattern of p16 in normal bladder mucosa, 15 normal bladder mucosa samples, along with cervical intraepithelial neoplasia tissue as a positive control, were used. p16 showed mild-to-moderate nuclear and cytoplasmic expression in all of the tissues of normal bladder mucosa (Fig. 1). Four different scoring methods were used for p16, named p16a, p16b, p16c, and p16d.

P16a: p16 nuclear staining percentage was scored between 0 and 3 as follows: no expression: score 0 , expression between 0 and 10\%: score 1, expression between $10 \%$ and $49 \%$ : score 2 , and strong expression, over $50 \%$ : score $3[8,17]$.

P16b: Expression below 10\% was regarded as abnormal while expression $\geq 10 \%$ was regarded as normal [18-20].

P16c: Expression below 5\% was regarded as abnormal while expression $\geq 5 \%$ was regarded as normal [1].

P16d: No expression of p16 (loss of p16 in tumor) or strong homogeneous expression over $50 \%$ (over expression) was regarded as abnormal and heterogeneous expression (below 50\%) was regarded as normal (Figs. 2C, 3C, 4C, 5C) [21-24].

While assessing scoring methods (p16a, p16b, p16c) the percentage of positive staining of tumor 

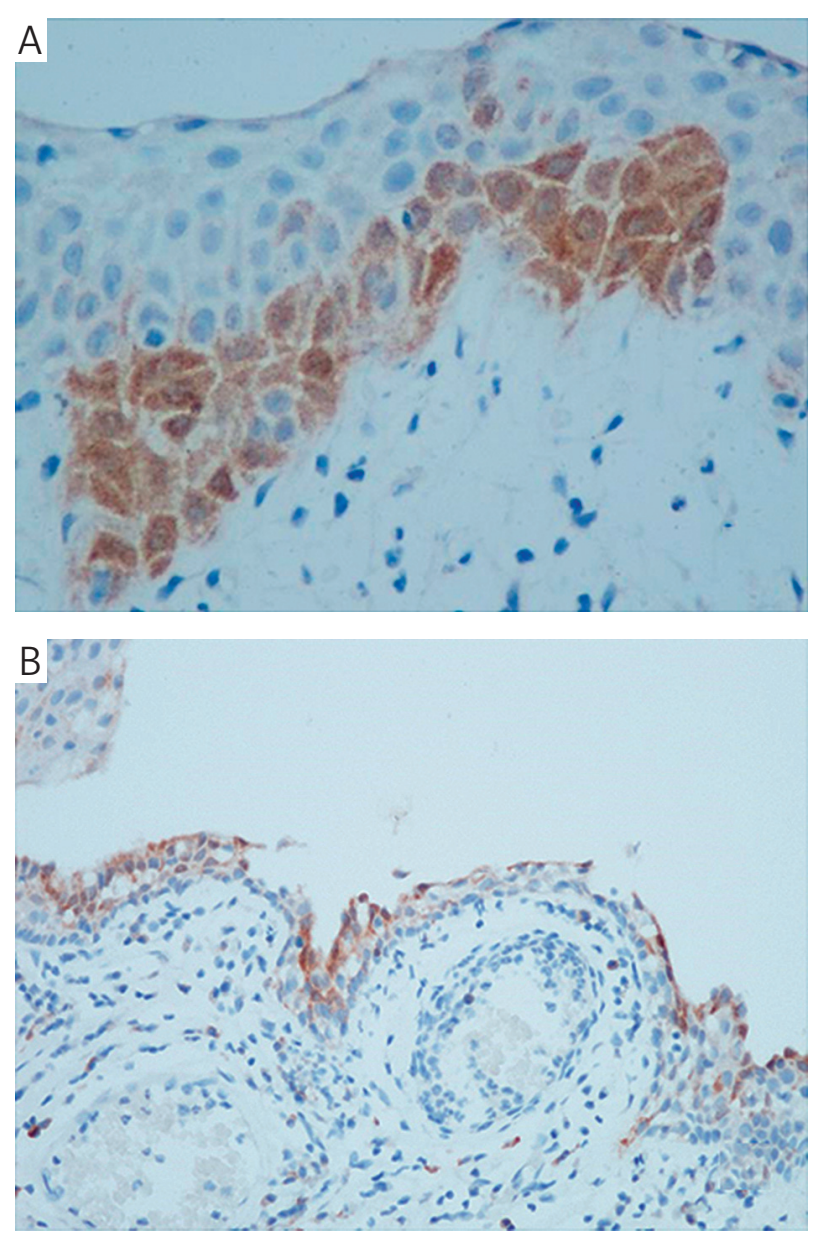

Fig. 1. A) p16 staining in normal transitional epithelium. Nuclear and cytoplasmic expression can be seen (p16, magnification $400 \times)$. B) p16 staining in normal transitional epithelium (p16, magnification $200 \times$ )

cells was calculated for each case regardless of homogeneous or heterogeneous stainings.

Tonsil tissue was used as a positive control for assessment of Ki-67 staining. While Ki-67 assessing maximum nuclear staining areas were choosen, we counted staining cells (average 4 high power field) and then set its percentage. The cut-off value was $10 \%$ for Ki-67. Tumors expressing below $10 \%$ were regarded negative while those expressing $\geq 10 \%$ were regarded as positive (Figs. 2B, 3B, 4B, 5B) [25].

For p16 and Ki-67 all reactive nuclei were considered positive irrespective of intensity.

\section{Statistical methods}

Statistical analyses were performed using IBM Statistical Package for the Social sciences (SPSS) Statistics for Windows, version 20.0 (IBM Corp., Armonk, NY). Continuous variables were tested for normality by the Kolmogorov-Smirnov test. Normally distributed data are presented as means \pm standard deviation. The rates and proportions of discrete variables were determined
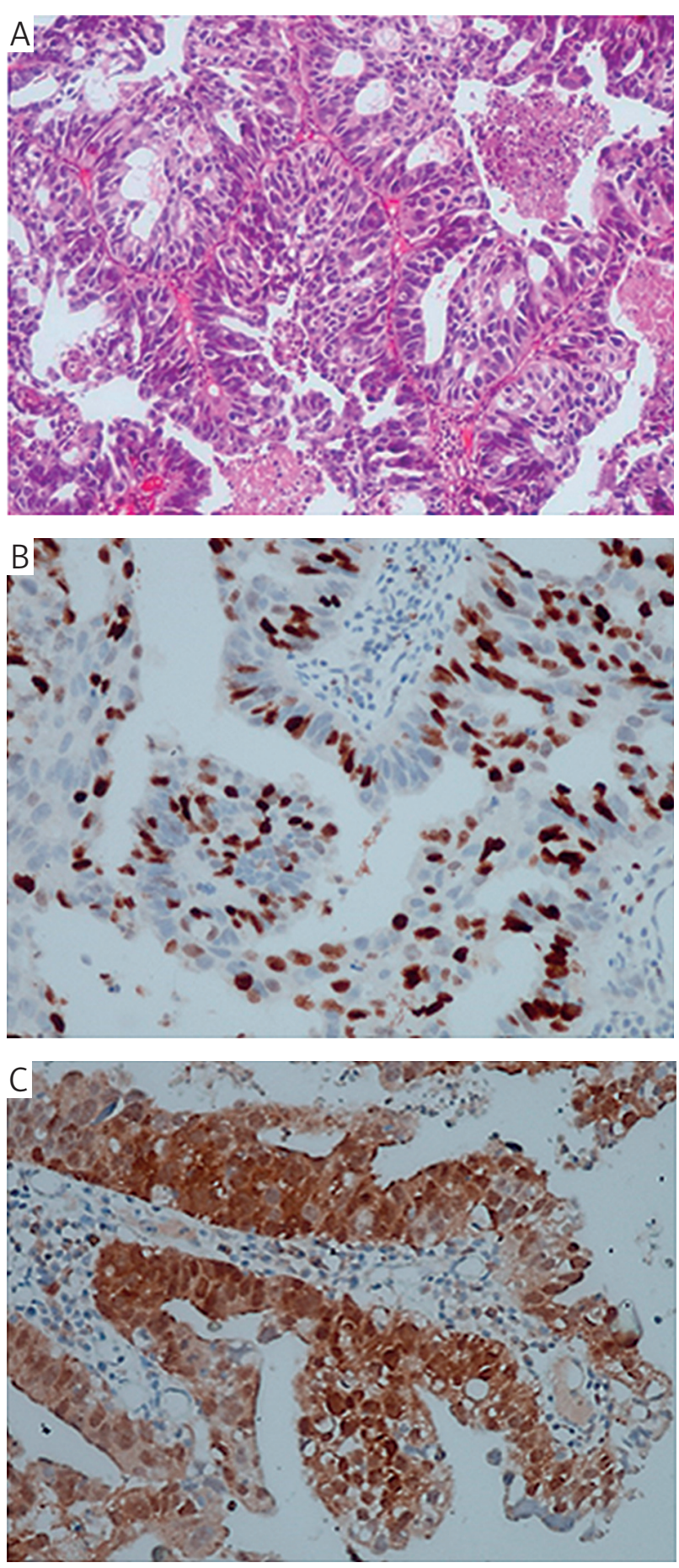

Fig. 2. A) High grade pT1 papillary urothelial carcinoma (HE, magnification $100 \times)$. B) High Ki-67 index (60\%) in the tumor (Ki-67, magnification $200 \times$ ). C) Abnormal expression of p16 (Scor 3) according to p16d scoring method. Tumor shows above $50 \%$ nuclear and cytoplasmic expression with p16 (p16, magnification $200 \times$ )

using the $\chi^{2}$. The median with data range (minimum to maximum) was used for non-normally distributed data. Correlations between tumor grade, stage and staining of $\mathrm{p} 16 \mathrm{~d}$ and $\mathrm{Ki}-67$ were evaluated using Spearman's rank correlation coefficient. Potential predictors of recurrence 

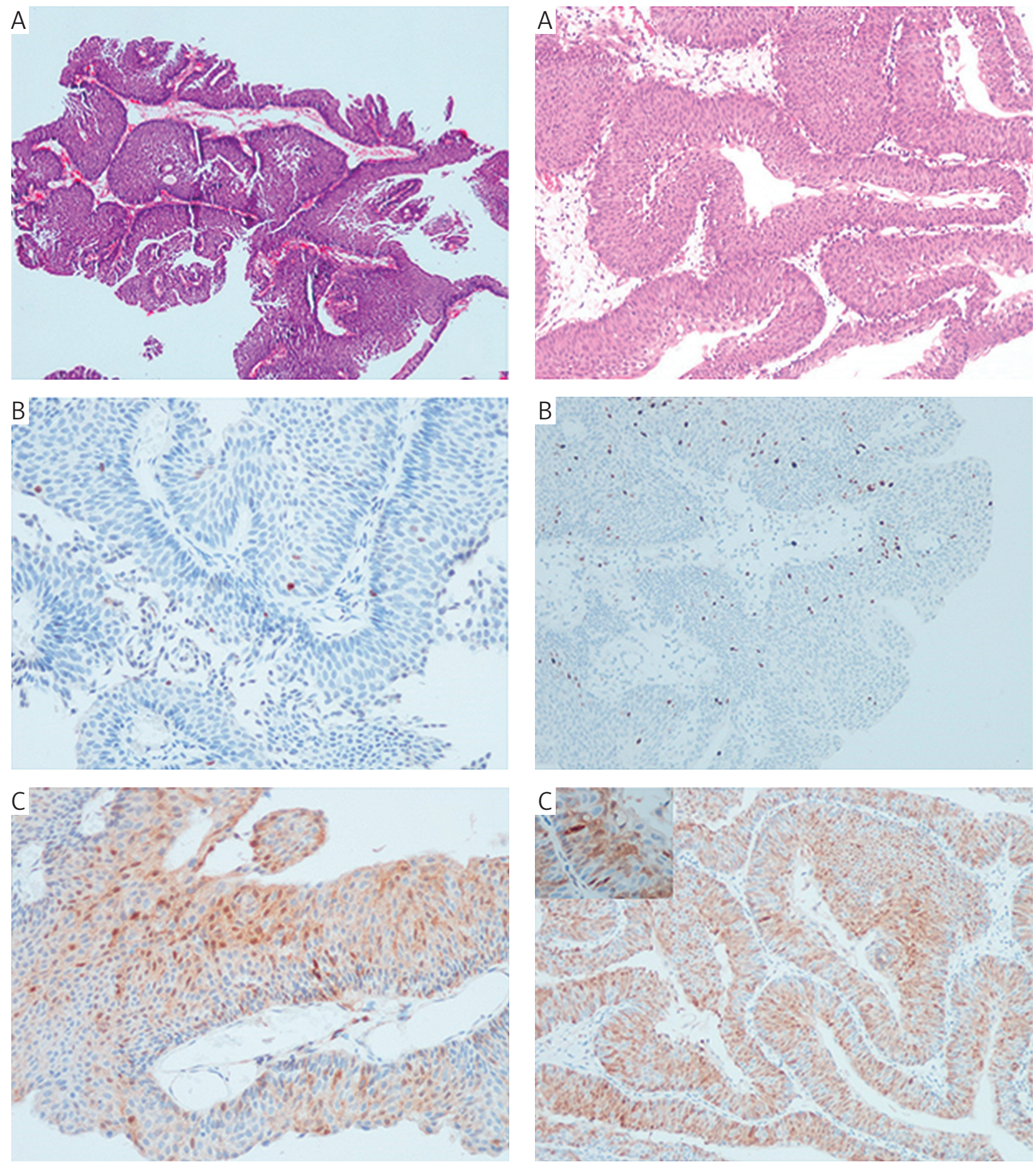

Fig. 3. A) Low grade pTa papillary urothelial carcinoma $(\mathrm{H} / \mathrm{E}$, magnification $40 \times)$. B) Low Ki-67 index $(1 \%)$ in the tumor (Ki-67, magnification $200 \times$ ). C) Normal p16 immunostaining (Scor 2) according to p16d scoring method. Tumor shows very low cytoplasmic expression but the nuclear staining is evident (p16, magnification $200 \times$ )

and progression in individual patients with superficial UC of the bladder were initially compared, and variables that showed a $\mathrm{p}$ value of $<$ 0.05 were included in a logistic regression model. Results were expressed as odds ratio (OR) and 95\% confidence interval (CI). The two-sided $\mathrm{p}$

Fig. 4. A) Low grade pT1 papillary urothelial carcinoma (HE, magnification $100 \times)$. B) Ki-67 index is below $10 \%(7 \%)$ in the tumor (Ki-67, magnification $100 \times$ ). C) Normal p16 staining (Scor 2) according to p16d scoring method. Nuclear staining is lower than cytoplasmic staining in the tumor (p16, magnification $100 \times$ ) (top left: p16, magnification $400 \times$ )

value of $<0.05$ was considered to indicate statistical significance. Relation between $\mathrm{Ki}-67$ and p16d staining with recurrence time was evaluated with log rank test. Recurrence rates were determined with Kaplan Meier analysis. A 5\% type-1 error level was used to infer statistical significance. 

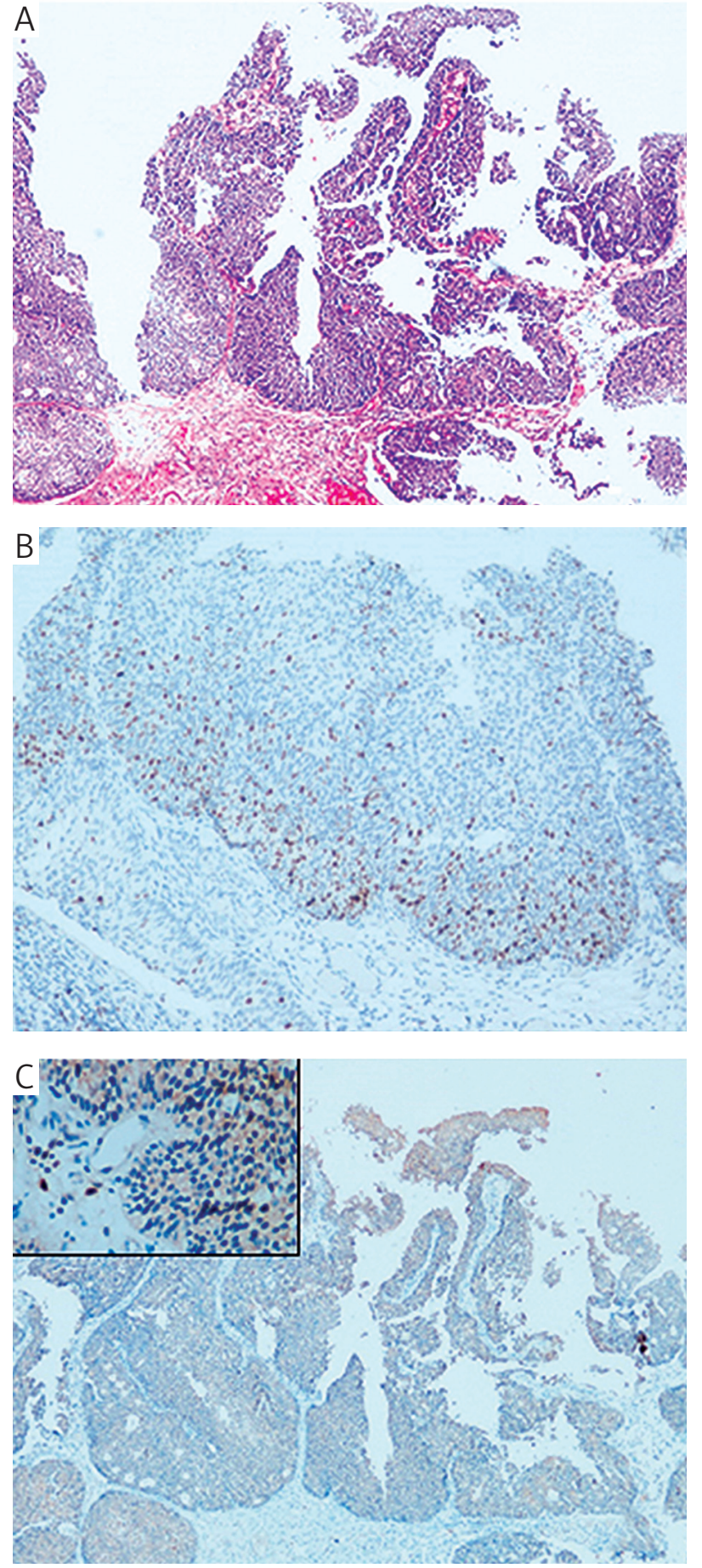

Fig. 5. A) Low grade pT1 papillary urothelial carcinoma (HE, magnification $40 \times$ ). $\mathrm{B}$ ) $\mathrm{Ki}-67$ index is above $10 \%$ $(17 \%)$ in the tumor $(\mathrm{Ki}-67$, magnification $100 \times)$. C) Abnormal expression of p16 (Scor 0). None of the tumor cells show p16d expression, but the positive p16 staining of fibroblasts can be seen as a positive internal control (p16, magnification $40 \times$ ) (top left: p16, magnification $400 \times$ )

\section{Results}

Of all the patients, 83 were males and 8 were females. The patients were divided into two groups based on recurrence and progression. The aver- age follow-up term was 32.8 (IQR 36.2-103.6) months.

Using Spearman's correlation test, Ki-67 was significantly positively correlated with tumor stage and tumor grade $(r=0.372$ and 0.443 , respectively; $\mathrm{p}<0.001)$. Abnormal p16d staining was also significantly positively correlated both with tumor stage and tumor grade $(r=0.307, p=0.003$ and $r=0.279$, $p=0.008$, respectively). There wasn't any statistically significant correlation between the other staining pattern of p16 (p16a, p16b, p16c) and tumor gradestage. In the recurrence group, abnormal p16d staining was observed in $28(45.2 \%)$ patients. There was a statistically significant between relation recurrence and abnormal p16d staining $(\mathrm{p}=0.005)$. However, there was no significant relation between abnormal p16d staining and progression $(\mathrm{p}=0.183)$. Regarding the other p16 staining patterns (p16a, $\mathrm{p} 16 \mathrm{~b}, \mathrm{p} 16 \mathrm{c}$ ), there was no statistically significant difference in terms of recurrence or progression.

In the recurrence group, $\mathrm{Ki}-67$ was $<10$ in $12(19.4 \%)$ patients and $\geq 10$ in $50(80.6 \%)$ patients. However, no such difference was detected in the progression group $(p=0.374)$. In the group having both $\mathrm{Ki}-67 \geq 10$ and abnormal p16d staining, $22(81.5 \%)$ patients showed recurrence while in the patient group having both Ki-67 $<10$ and normal p16d staining, $5(18.5 \%)$ patients had recurrence. There was a statistically significant difference in favor of the first group $(p<0.001)$. However, there was no difference in terms of progression ( $\mathrm{p}=0.126)$. In the group with $\mathrm{Ki}-67 \geq 10$ and normal p16d staining, there were 29 (56.9\%) patients. When compared with the patient group with $\mathrm{Ki}-67 \geq 10$ and abnormal p16d, there was a statistically significant difference in favor of this group ( $\mathrm{p}=0.035$, I).

In the recurrence group, the tumor size was $<3 \mathrm{~cm}$ in $24(39.3 \%)$ patients and $\geq 3 \mathrm{~cm}$ in $37(60.7 \%)$. A statistically significant difference was found between recurrence and tumor size $(\mathrm{p}<0.001)$. In the progression group, the tumor size was $<3 \mathrm{~cm}$ in $5(31.2 \%)$ patients and $\geq 3 \mathrm{~cm}$ in $11(68.8 \%)$. However, there was no statistically significant difference between progression and tumor size $(\mathrm{p}=0.071$, Table I). Recurrence rate was significantly higher in patients with multiple tumors than in those with a single tumor $(51.6 \%$ vs. $48.4 \%, p=0.003)$. There was also a positive correlation between tumor number and progression $(r=0.320$, $p=0.002)$. The progression rate was significantly higher in patients with multiple tumors than in patients with a single tumor ( $75 \%$ vs. $25 \%$, respectively, $p=0.002)$. Similarly, Spearman's correlation test revealed that the stage and grade of tumor were positively correlated with recurrence $(\mathrm{r}=0.253, \mathrm{p}=0.016$ and $r=0.288, p=0.006$, respectively). In the recurrence group, $23(37.1 \%)$ patients were pTa and $39(62.9 \%)$ were pT1. There was a statistically sig- 
Table I. Comparison of patients according to presence of recurrence and progression

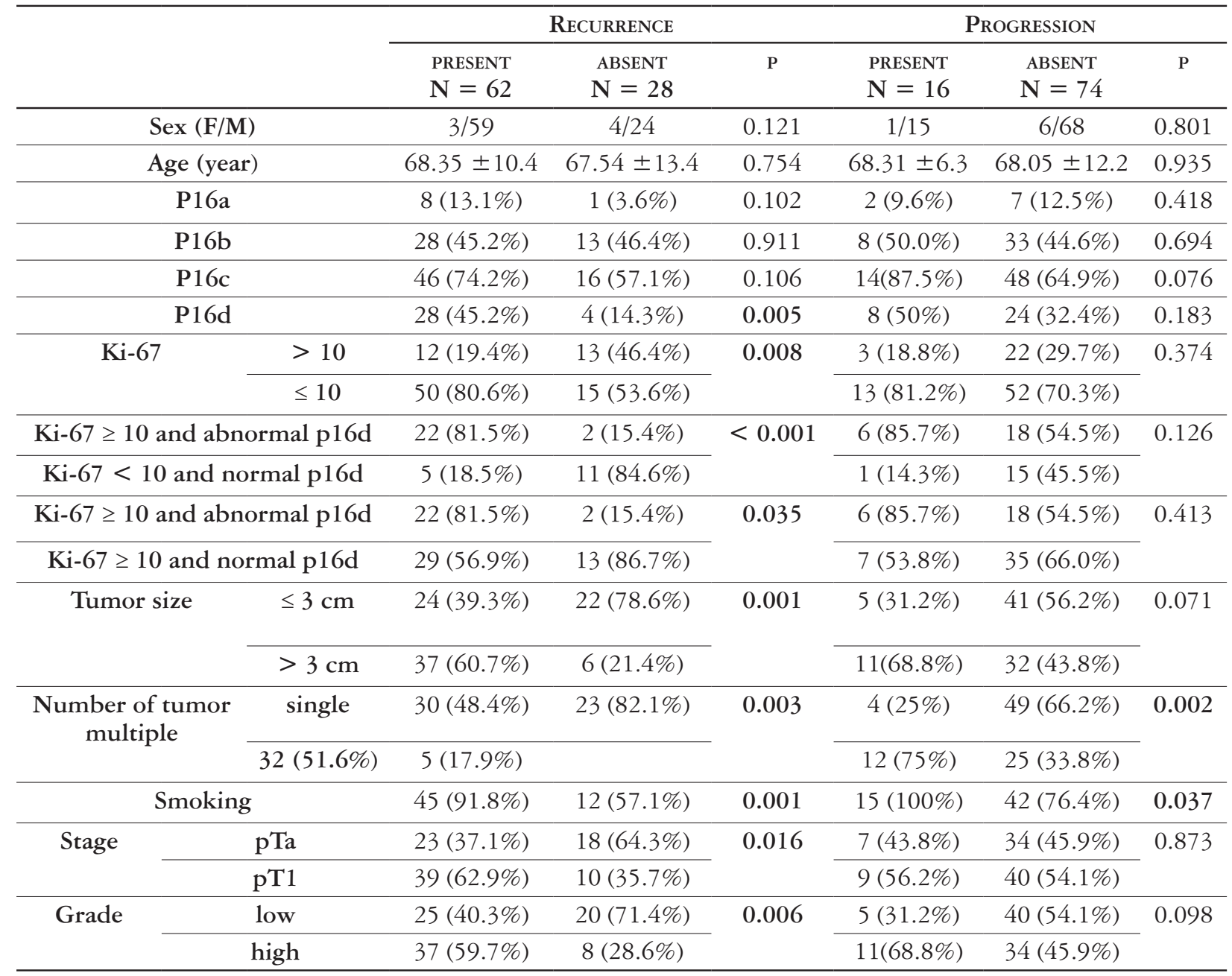

Table II. Multivariate logistic regression analysis of independent predictive factors for recurrence

\begin{tabular}{ccccc}
\hline & & & Multivariate ANALYsis \\
\hline & ReFERENCE & OR & 95\% CI & P \\
\hline Smoking & & 1.27 & $(0.66-18.99)$ & 0.139 \\
\hline Tumor size & $\geq 3 \mathrm{~cm}$ & 0.85 & $(0.54-10.2)$ & 0.257 \\
\hline Stage & $\mathrm{pT} 1$ & 0.71 & $(0.23-3.78)$ & 0.924 \\
\hline Ki-67 & $\geq 10$ & 1.79 & $(1.01-33.56)$ & 0.062 \\
\hline P16d & abnormal staining & 2.14 & $(0.58-12.1)$ & 0.118 \\
\hline Ki-67 $\geq 10$ and abnormal p16d & & 2.26 & $(0.13-46.41)$ & 0.035 \\
\hline
\end{tabular}

nificant difference between stage and recurrence $(\mathrm{p}=0.016)$. In the recurrence group, $25(40.3 \%) \mathrm{pa}-$ tients were low grade and $37(59.7 \%)$ patients were high grade. There was a statistically significant difference between grade and recurrence $(p=0.006)$. Similarly, there was a significant difference between smoking and both recurrence and progression $(p<0.001$ and $p=0.037$, respectively, Table I).

According to the univariate analysis, abnormal p16d staining, Ki-67 $\geq 10$, tumor size $\geq 3 \mathrm{~cm}$, multiple tumors, smoking, pT1 stage, and high-grade tumor were definite risk factors for recurrence. As there was a significant correlation between tumor number and size and between tumor grade and stage, only tumor size and stage were evaluated in the multivariate analysis. In the multivariate logistic regression analysis, combined $\mathrm{Ki}-67 \geq 10$ and abnormal p16d staining was found to be the only independent predictive factor for recurrence $(\mathrm{OR}=2.26,95 \% \mathrm{CI}: 0.13-46.41, \mathrm{p}=0.035)$ and no independent predictive factor for progression was found (Table II). 
A

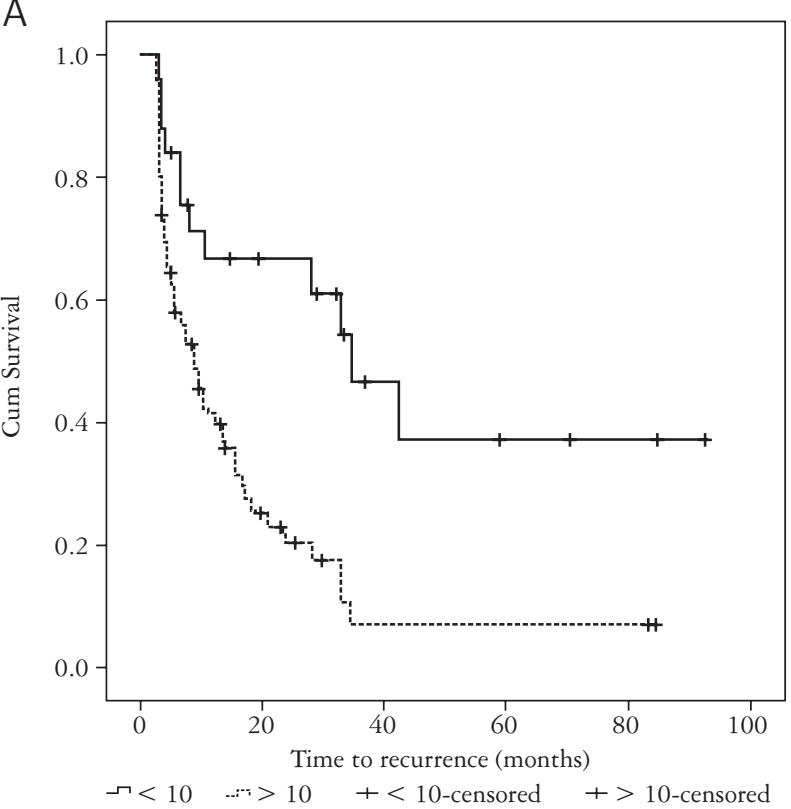

B

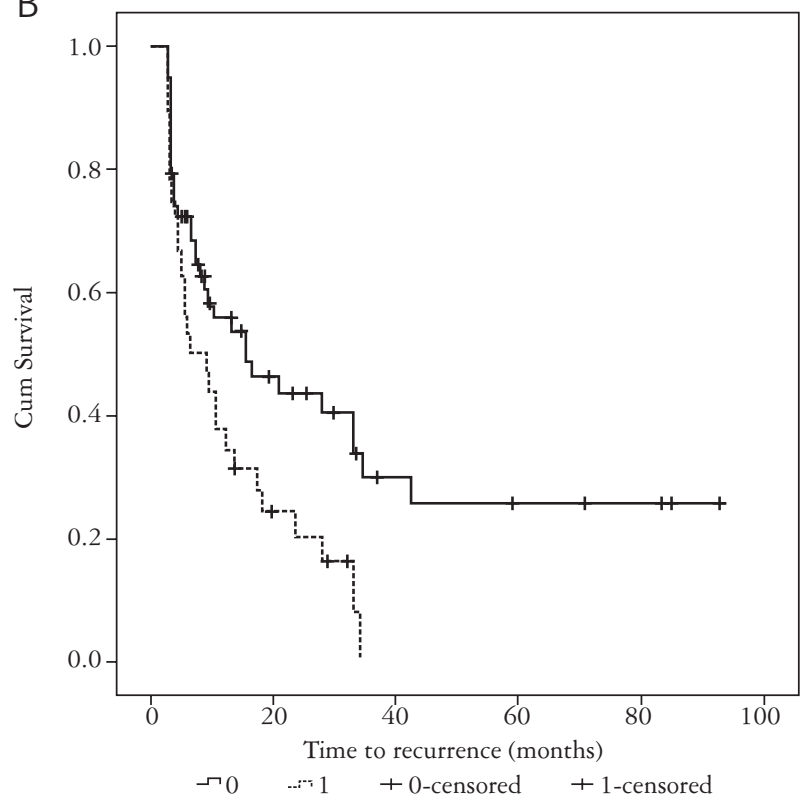

Fig. 6. A) The relationship between with $\mathrm{Ki}-67$ and recurrence time. B) The relationship between with p16 and recurrence time

Based on a Kaplan-Meier analysis, the median time to recurrence was 34.7 (95\% CI: 20.4-49) months in the group in which Ki-67 staining was $\geq 10$, and 9.1 (95\% CI: 5.5-12.7) months in the group where $\mathrm{Ki}-67$ staining was $<10$. In terms of median time to recurrence, there was a statistically significant difference between the two groups $(p<0.001$, Fig. 6A).

Based on this analysis, the median time to recurrence was 6.6 (95\% CI: 1.2-11.9) months in the group with abnormal p16d staining and 15.6 (95\% CI: 3.6-27.6) months in the group with normal p16d staining. In terms of median time to recurrence, there was a statistically significant difference between the group with abnormal p16d staining and that with normal p16d staining ( $\mathrm{p}=0.015$, Fig. 6B).

\section{Discussion}

Many studies have shown that Ki-67 and p16 staining are independent immunohistochemical markers predictive of recurrence and progression in bladder cancer $[15,19,20,26,27]$. As with many tissue section studies, there are various approaches to immunohistochemical evaluation of p16 in the bladder. p16, which is more difficult to evaluate than cell cycle proteins, such as $\mathrm{Ki}-67$ and p53, is a valuable marker for predicting the clinical course $[1,19]$.

The p16 protein is a component of the p16/cyclin $\mathrm{D} 1 / \mathrm{Rb}$ pathway and controls the G1-S transition of the cell cycle. In most tumors, the control of G1 progression and initiation of $\mathrm{S}$ phase have deteriorated, which causes unlimited entry into the cell cycle and cell proliferation [28]. It has been shown that abnormal expression of p16 is associated with several cancers, such as lung, laryngeal, and tonsillary carcinomas [29-31]. Various scoring methods have been used in studies comparing p16 expression and prognostic parameters in various neoplasms [32-34]. In bladder cancer, there is no consensus about the regarding the p16 staining pattern.

In some of these studies, p16 was evaluated using a score of 0 to 3 (no staining: score 0 , staining between 0 and 10\%: score 1 , staining between 10 and $49 \%$ : score 2, strong staining: score 3) [8, 17]. In other studies, expression below $10 \%$ was regarded as abnormal, while that above $10 \%$ was considered normal [18-20]. Another study interpreted p16 expression below $5 \%$ as negative staining for p16 [1]. Some authors regarded expression loss (no staining) and overexpression (staining $>50 \%$ ) as abnormal and moderate staining as normal [21-24]. Four of the scoring methods employed in these studies were also used in our work for comparison.

p16 is a marker expressed in both the nucleus and cytoplasm. However, the functional and biological importance of cytoplasmic staining is not yet understood [35]. Some studies on p16 expression take into account only nuclear staining [1, 24, 36], while others consider both nuclear and cytoplasmic staining [18, 19, 23]. Han et al. compared endocervical carcinomas and endometrial adenocarcinomas in terms of p16 expression. Use of nuclear staining only as a reference for scoring was claimed to be the most adequate and effective method [35]. Similarly, in a study evaluating p16 expression in ovarian cancer, Kommoss et al. took into consideration only nuclear staining in scoring [36]. In our study, p16 
expression of all tumor tissues was scored based on the four abovementioned staining patterns by taking only nuclear staining as a reference. The results obtained were assessed in terms of predicting recurrence and progression in superficial bladder tumors.

Krüger et al. used a tissue microarray (TMA) technique and used p16b as a reference for scoring (expression $<10 \%$ was regarded as abnormal and expression $\geq 10 \%$ as normal) and found a significant correlation between p16 and progression; however, staining did not correlate with recurrence [19]. However, we found no statistically significant correlation between $\mathrm{p} 16 \mathrm{~d}$ and progression, likely due to the limited number of patients showing progression. In our study, there was a statistically significant association between recurrence and p16 expression only when the p16d scoring system was used. Olsson et al. [23] also used the same staining pattern (p16d) in stage T1 urinary bladder cancers and they detected normal p16 expression was related to a lower risk of tumor progression. Similar to our study, Lee et al. [21] investigated four cell cycle proteins (p16, pRb, $\mathrm{p} 53$, cyclin D1) and found no correlation between progression and p16 (p16d method) expression; their results suggested that multiple genetic defects affected the clinical course and metastatic capacity of bladder carcinomas. Shariat et al used the staining pattern of p16d and they determined that combined staining of p16 and $\mathrm{pRb}$ can be a useful marker in prognosis of bladder cancer [22]. In our study we detected that evaluating p16d and $\mathrm{Ki}-67$ in combination was an independent predictor for recurrence in $\mathrm{pTa}$ and $\mathrm{pT} 1$ bladder cancers. In another study in which p16 staining $<10 \%$ was regarded as abnormal (p16b), a significant correlation was found between p16 expression and recurrence, as in our work, but there was no correlation between p16 and progression [20].

Some of previous studies investigated the association of Human papillomavirus (HPV) and p16 expression in UC. Steinestel et al. research on p16(INK4a) immunoexpression followed by detection and subclassification of HPV DNA in total of 45 patients (UC in situ and controls). They didnt't find any significant correlation between HPV and p16 overexpression in UC in situ [37]. In a different study that analyzed HPV and p16 relationship in UC with squamous differentiation, they claimed p16 expression did not appear to be a strong representative marker for evidence of HPV infection in this type of cancer [38].

$\mathrm{Ki}-67$ is expressed throughout the cell cycle, with the exception of the $G_{0}$ phase, and shows proliferative activity in various carcinomas [39]. In our study, there was no significant correlation between Ki-67 and progression, while a significant correlation was found between recurrence and $\mathrm{Ki}-67$ expression.
Similar to our results, Mhawech et al. classified pT1 tumors and stated that $\mathrm{Ki}-67$ had no importance in predicting progression [25]. In a study of $226 \mathrm{pa}$ tients with bladder-confined cystectomy, Margulis et al. found a significant correlation between recurrence and $\mathrm{Ki}-67$ staining, consistent with our results [14]. In 332 patients with bladder cancer, Ding et al. found that $\mathrm{Ki}-67$ expression was an independent predictor of both recurrence and progression [15].

We found a statistically significant correlation between recurrence and cases with both abnormal p16d staining and $\mathrm{Ki}-67 \geq 10 \%$. This result was beyond the ability of Ki-67 and p16d individually to predict recurrence. In a study of pTa and $\mathrm{pT} 1$ tumors in the bladder, Hitchings et al. found a statistically significant correlation between abnormal expression of both $\mathrm{p} 53$ and $\mathrm{p} 16$ and progression [18]. Korkolopoulou et al. also indicated that p53/p16 combined expression was an independent predictor of decreased survival in muscle-invasive tumours in bladder cancers [40]. Additionally with the conclusion of these previous studies our results suggested that using the combination of $\mathrm{p} 16 \mathrm{~d} / \mathrm{Ki} 67$ markers have more predictive power than either marker alone for recurrence.

\section{Conclusions}

Although several studies have investigated the roles of $\mathrm{p} 16$ and $\mathrm{Ki}-67$ expression in predicting prognosis in bladder carcinomas, there is still no consensus regarding the most appropriate method of scoring p16 expression. In our study, four different p16 expression scoring methods in bladder carcinomas were compared. We believe that p16d scoring system, which used normal transitional epithelial staining pattern as a reference, is most objective method for predicting bladder tumors recurrence and progression. Moreover, in our study, it was found that evaluating p16d and $\mathrm{Ki}-67$ in combination could facilitate prediction of recurrence in superficial bladder tumors. Further studies of p16 expression patterns in a larger series of patients, including a greater number with progression and employing other cell cycle proteins in combination, are needed.

The authors declare no conflict of interest.

\section{References}

1. Yang CC, Chu KC, Chen HY, Chen WC. Expression of p16 and cyclin D1 in bladder cancer and correlation in cancer progression. Urol Int 2002; 69: 190-194.

2. Kirkali Z, Chan T, Manoharan M, et al. Bladder cancer: epidemiology, staging and grading, and diagnosis. Urology 2005; 66 (6 Suppl 1): 4-34 
3. Babjuk M, Oosterlinck W, Sylvester R, et al. EAU guidelines on non-muscle-invasive urothelial carcinoma of the bladder. Eur Urol 2008; 54: 303-314.

4. Sylvester RJ, van der Meijden AP, Oosterlinck W, et al. Predicting recurrence and progression in individual patients with stage Ta T1 bladder cancer using EORTC risk tables: a combined analysis of 2596 patients from seven EORTC trials. Eur Urol 2006; 49: 466-465; discussion 475-477.

5. Dodurga Y, Avcı CB, Yılmaz S, et al. UroVysion fluorescence in situ hybridization (UroVysion FISH) assay for detection of bladder cancer in voided urine of Turkish patients: a preliminary study. Contemp Oncol (Pozn) 2013; 17: 156-160.

6. Poletajew S, Fus $\mathrm{E}$, Walędziak M, et al. Comparison of pathological staging and grading of urothelial bladder carcinoma in post-transurethral resection and post-radical cystectomy specimens. Pol J Pathol 2014; 65: 305-312.

7. Goyal S, Singh UR, Sharma S, Kaur N. Correlation of mitotic indices, $\mathrm{AgNor}$ count, $\mathrm{Ki}-67$ and $\mathrm{Bcl}-2$ with grade and stage in papillary urothelial bladder cancer. Urol J 2014; 11: 1238-1247.

8. Raspollini MR, Minervini A, Lapini A, et al. A proposed score for assessing progression in $\mathrm{pT} 1$ high-grade urothelial carcinoma of the bladder. Appl Immunohistochem Mol Morphol 2013; 21: 218-227.

9. Salehinejad J, Sharifi N, Amirchaghmaghi M, et al. Immunohistochemical expression of p16 protein in oral squamous cell carcinoma and lichen planus. Ann Diagn Pathol 2014; 18: 210-213.

10. Rawish KR, Desouki MM, Crispens MA, Fadare O. Conventional endometrioid adenocarcinomas of the endometrium recurring as clear cell tumors: comparative immunohistochemical analyses. Ann Diagn Pathol 2013; 17: 270-275.

11. van Bogaert LJ. P16INK4a immunocytochemistry/immunohistochemistry: need for scoring uniformization to be clinically useful in gynecological pathology. Ann Diagn Pathol 2012; 16: $422-426$.

12. Chatterjee SJ, George B, Goebell PJ, et al. Hyperphosphorylation of $\mathrm{pRb}$ : a mechanism for $\mathrm{RB}$ tumour suppressor pathway inactivation in bladder cancer. J Pathol 2004; 203: 762-770.

13. Sarkar S, Jülicher KP, Burger MS, et al. Different combinations of genetic/epigenetic alterations inactivate the $\mathrm{p} 53$ and $\mathrm{pRb}$ pathways in invasive human bladder cancers. Cancer Res 2000; 60: 3862-3871.

14. Margulis V, Shariat SF, Ashfaq R, et al. Ki-67 is an independent predictor of bladder cancer outcome in patients treated with radical cystectomy for organ-confined disease. Clin Cancer Res 2006; 12: 7369-7373.

15. Ding W, Gou Y, Sun C, et al. Ki-67 is an independent indicator in non-muscle invasive bladder cancer (NMIBC); combination of EORTC risk scores and $\mathrm{Ki}-67$ expression could improve the risk stratification of NMIBC. Urol Oncol 2014; 32: 42.e13-9.

16. Pich A, Chiusa L, Formiconi A, et al. Proliferative activity is the most significant predictor of recurrence in noninvasive papillary urothelial neoplasms of low malignant potential and grade 1 papillary carcinomas of the bladder. Cancer 2002; 95: 784-790.

17. Rajcani J, Kajo K, Adamkov M, et al. Immunohistochemical characterization of urothelial carcinoma. Bratisl Lek Listy 2013; 114: 431-438.

18. Hitchings AW, Kumar M, Jordan S, et al. Prediction of progression in pTa and pT1 bladder carcinomas with p 53, p16 and pRb. Br J Cancer 2004; 91: 552-557.

19. Krüger S, Mahnken A, Kausch I, Feller AC. P16 immunoreactivity is an independent predictor of tumor progression in minimally invasive urothelial bladder carcinoma. Eur Urol 2005; 47: 463-467.

20. Bartoletti R, Cai T, Nesi G, et al. Loss of P16 expression and chromosome 9p21 LOH in predicting outcome of patients affected by superficial bladder cancer. J Surg Res 2007; 143: 422-427.
21. Lee K, Jung ES, Choi YJ, et al. Expression of pRb, p53, p16 and cyclin D1 and their clinical implications in urothelial carcinoma. J Korean Med Sci 2010; 25: 1449-1455.

22. Shariat SF, Tokunaga H, Zhou J, et al. p53, p21, pRB, and p16 expression predict clinical outcome in cystectomy with bladder cancer. J Clin Oncol 2004; 22: 1014-1024.

23. Olsson H, Hultman P, Monsef N, et al. Immunohistochemical evaluation of cell cycle regulators: impact on predicting prognosis in stage t1 urinary bladder cancer. ISRN Urol 2012; 2012: 379081 .

24. Benedict WF, Lerner SP, Zhou J, et al. Level of retinoblastoma protein expression correlates with p16 (MTS-1/INK4A/CDKN2) status in bladder cancer. Oncogene 1999; 18: 1197-1203.

25. Mhawech P, Greloz V, Oppikofer C, et al. Expression of cell cycle proteins in T1a and T1b urothelial bladder carcinoma and their value in predicting tumor progression. Cancer 2004; 100: 2367-2375.

26. Santos LL, Amaro T, Pereira SA, et al. Expression of cell-cycle regulatory proteins and their prognostic value in superficial low-grade urothelial cell carcinoma of the bladder. Eur J Surg Oncol 2003; 29: 74-80.

27. Yurakh AO, Ramos D, Calabuig-Fariñas S, et al. Molecular and immunohistochemical analysis of the prognostic value of cell-cycle regulators in urothelial neoplasms of the bladder. Eur Urol 2006; 50: 506-515; discussion 515.

28. Patrick DL, Althof SE, Pryor JL, et al. Premature ejaculation: an observational study of men and their partners. J Sex Med 2005; 2: 358-367.

29. Igarashi T, Jiang SX, Kameya T, et al. Divergent cyclin B1 expression and Rb/p16/cyclin D1 pathway aberrations among pulmonary neuroendocrine tumors. Mod Pathol 2004; 17: $1259-1267$

30. Li D, Zhang Q, Zhang X. Expression and clinical significance of MDM2 and P16 in laryngeal squamous cell carcinoma. Lin Chung Er Bi Yan Hou Tou Jing Wai Ke Za Zhi 2012; 26: 802805 .

31. Mellin Dahlstrand H, Lindquist D, Björnestål L, et al. P16(IN$\mathrm{K} 4 \mathrm{a}$ ) correlates to human papillomavirus presence, response to radiotherapy and clinical outcome in tonsillar carcinoma. Anticancer Res 2005; 25: 4375-4383.

32. Alshenawy HA. Evaluation of p16, human papillomavirus capsid protein $\mathrm{L} 1$ and $\mathrm{Ki}-67$ in cervical intraepithelial lesions: Potential utility in diagnosis and prognosis. Pathol Res Pract 2014; 210: 916-921.

33. Cerski MR, Pereira F, Matte US, et al. Exon 11 mutations, Ki67, and p16(INK4A) as predictors of prognosis in patients with GIST. Pathol Res Pract 2011; 207: 701-706.

34. Terzi A, Saglam EA, Barak A, Soylemezoglu F. The significance of immunohistochemical expression of Ki-67, p53, p21, and p16 in meningiomas tissue arrays. Pathol Res Pract 2008; 204: 305-314.

35. Han CP, Kok LF, Wang PH, et al. Scoring of p16(INK4a) immunohistochemistry based on independent nuclear staining alone can sufficiently distinguish between endocervical and endometrial adenocarcinomas in a tissue microarray study. Mod Pathol 2009; 22: 797-806.

36. Kommoss S, du Bois A, Ridder R, et al. Independent prognostic significance of cell cycle regulator proteins p16(INK4a) and $\mathrm{pRb}$ in advanced-stage ovarian carcinoma including optimally debulked patients: a translational research subprotocol of a randomised study of the Arbeitsgemeinschaft Gynaekologische Onkologie Ovarian Cancer Study Group. Br J Cancer 2007; 96: 306-313

37. Steinestel J, Cronauer MV, Müller J, et al. Overexpression of p16(INK4a) in urothelial carcinoma in situ is a marker for MAPK-mediated epithelial-mesenchymal transition but is not related to human papillomavirus infection. PLoS One 2013; 8: e65189. 
38. Kim SH, Joung JY, Chung J, et al. Detection of human papillomavirus infection and p16 immunohistochemistry expression in bladder cancer with squamous differentiation. PLoS One 2014; 9: e93525.

39. Vorreuther R, Hake R, Borchmann P, et al. Expression of immunohistochemical markers (PCNA, Ki-67, 486p and p53) on paraffin sections and their relation to the recurrence rate of superficial bladder tumors. Urol Int 1997; 59: 88-94.

40. Korkolopoulou P, Christodoulou P, Lazaris A, et al. Prognostic implications of aberrations in $\mathrm{p} 16 / \mathrm{pRb}$ pathway in urothelial bladder carcinomas: a multivariate analysis including p53 expression and proliferation markers. Eur Urol 2001; 39: 167-177.

\section{Address for correspondence}

Gülzade Özyalvaçlı MD

Department of Pathology, Faculty of Medicine

Abant Izzet Baysal University

14280 Bolu, Turkey

tel. +90 5053911468 , fax +903742534515

e-mail: gulzade78@yahoo.com 